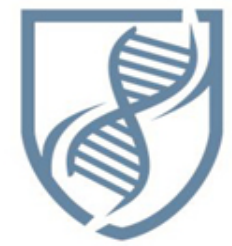

Journal of Bioscience and Applied Research

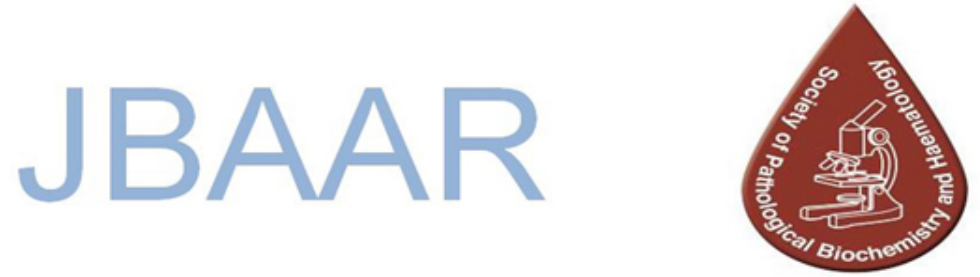

WWW.JBAAR.ORG

\title{
Gene evolution and diversity of living organisms theory
}

\author{
Sobhy E. Hassab El-Nabi \\ Zoology Department, Faculty of Science, Menufia University, Egypt \\ (Corresponding author e.mail.
}

\begin{abstract}
Statement of the theory is \{Evolution of organisms depends mainly on gene evolution through the appearance of novel genes. Diversity of Living organisms results from genetic variations. Each Individual in the same species of Living Organisms has its own Identity of DNA Finger-print. Variations of Individuals Results from Effect of Environmental Factors, Mutation and Invader of Genetic Materials\}. Gene evolution depends mainly on gene duplication ,variation of environmental conditions, mutation, transposable elements, horizontal gene transfer (HGT), de nove gene that originated from non-coding DNA which mainly comes from retrovirus and micro-RNA. And invader nucleic acids from viruses, bacteria or any parasite.The main prediction of this theory is not only each individual of human being has unique DNA fingerprint, but also any individual in the same species has also unique identity in DNA fingerprint. Any living organisms acquire genetic materials through its intimate contacts with another organisms . If the acquired genetic material reach to germ cells, it could transfer to next generation giving new traits.Viruses considered the main source for genetic transformation in all living organisms. The genomic materials of some viruses could integrate inside human genome like hepatitis B and HIV. The human genome has about $5 \%$ virus genetic material especially from retroviruses.This theory could explain the appearance of new individuals according to transfer of genetic materials during intimate contact between different species, and support many researchers who discovered new species. Also ,this theory explain and will answer different questions, why husband and wife after some time, nearly acquire some similarities ?, why people in the same geographical area nearly have a similar pattern ?. The answer on this questions is return to the transfer of micro RNA which found in food, fluids and environment that

surround the organisms. Also, similarity may return to transfer of transposable elements through intimate contacts within individuals. This theory also, explain the hostparasite relationship depending through transfer of DNA and RNA between them. We developed in our lab. Simple technique for detection of DNA and RNA from tissue lysate, through it micro- RNA could be detected. We detected the instability and dynamicity of DNA through sister chromatide exchange (SCE). Also we detect individual variation in the same species through haplotype analysis, this support our theory.

\section{Key words: Gene evolution, Diversity, Mutation, DNA}

\section{Previous works support the proposed theory:}

Theory of evolution and diversity of living organisms is not only explained on the morphological character (Phenotype), but also on genetic character (genotype) through gene evolution. The flow of genetic information start from gene through transcription process that produce mRNA which translate to protein on ribosomes through translation process (Fig. 1).

Several mechanisms frequently cooperate in generating a new gene (Fig. 2) through exon or domain shuffling, gene duplication, retrotransposition, transposen elements (TE) domestication, lateral gene transfer, gene fission or fusion, de novo origination, reading -frame shift,alternative splicing, non-coding RNA and pseudogenes as RNA regulator.

DNA is responsible for the continuity of life due to its ability to have a replication character. If this molecule loses this character and the replication process stops, the biological life disappears on the earth, because cell division, growth, reproduction formation of embryos. Some genetic materials transfer in the same genome through
\end{abstract}




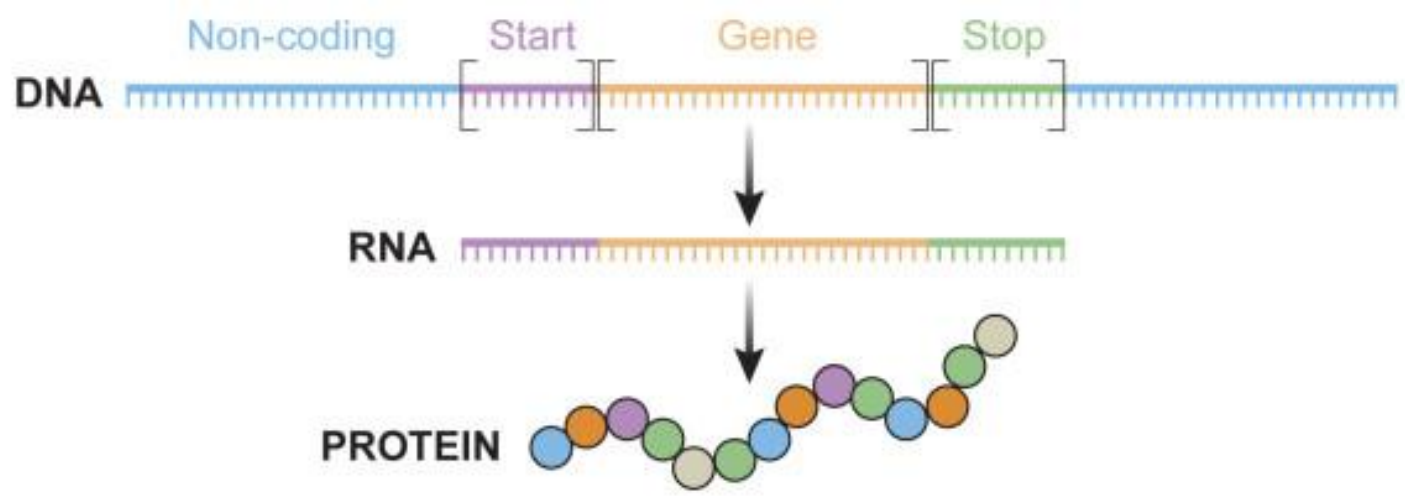

Fig. (1) The central dogma - DNA encodes RNA, which is turn is translated into proteins. Image@Edvotek 2014
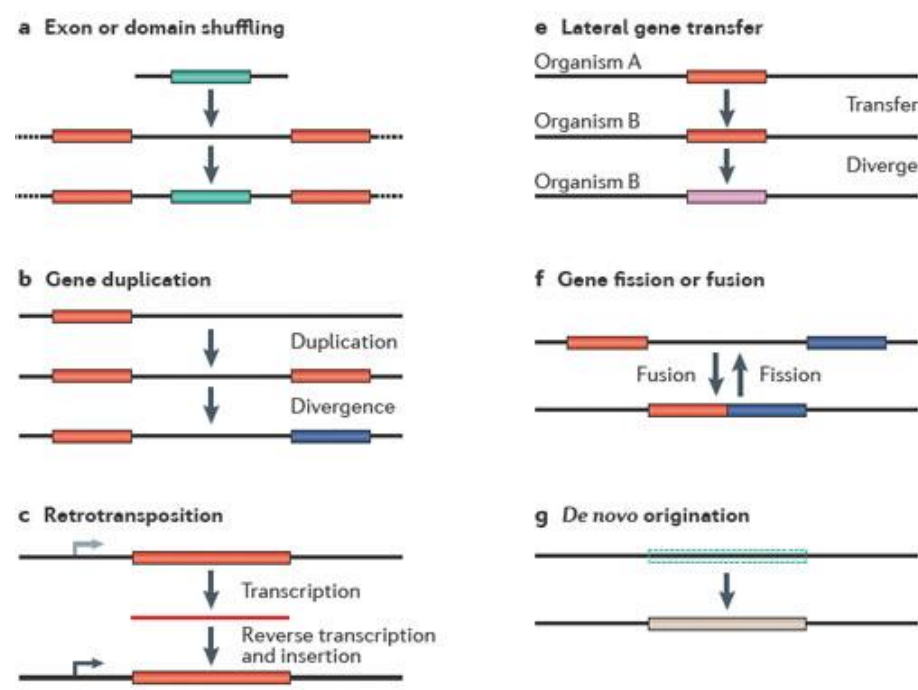

$f$ Gene fission or fusion

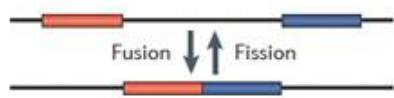

9 De novo origination

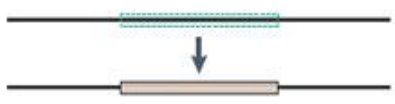

h Reading-frame shift

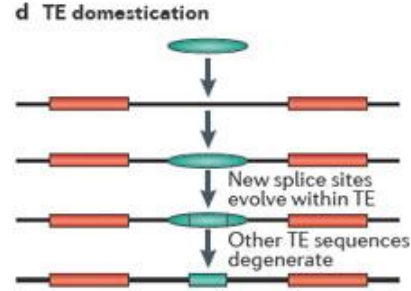

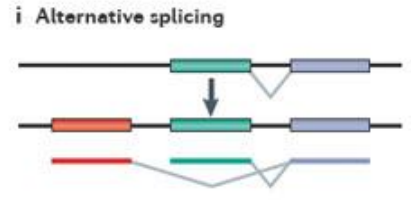

j Non-coding RNA
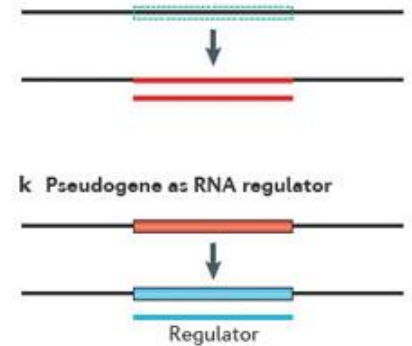

Fig. (2) Different mechanisms explain development of new genes (after Sidi et al., 2013)

transposable elements or from one organisms to another Understanding how genes originated and subsequently naturally through horizontal gene transfer (HGT) or evolve is crucial for explaining the genetic basis for the artificially through genetic engineering techniques forming origin and evolution of novel phenotypesand, ultimately, transforming organisms or transgenic animals. will biological diversity (Manyuan et al., 2013).Mutation of any stooped. DNA is considered as the book of the life for each genes(Fig. 3) in germ cell leads to formation of a protoorganism. Life is based on Identity and diversity, it start gene. This proto-gene structure must then spread through with identity then gene evolution leads to diversity, then the population until it is fixed. To form new gene, about 5 return back to identity for each new born individual. mechanisms were done. 


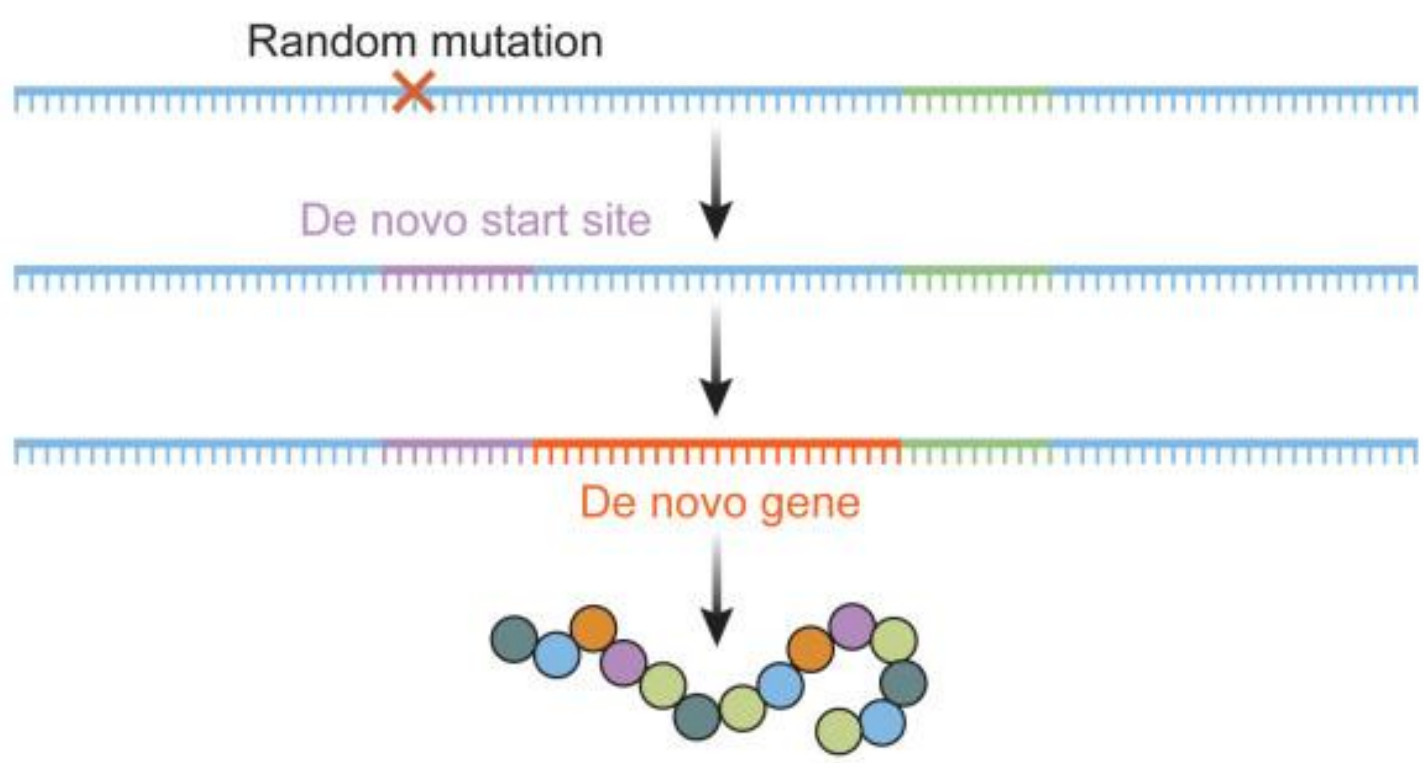

Fig. (3)The de novo formation of a gene. A random mutation leads to the creation of a new start site within a non-coding region of DNA, resulting in the formation of a new gene. Image (CEdvotek 2014.

The first one is gene duplicationwhich is thought to contribute most to the generation of new genes. A single or few new gene structure(s) can be formed at one time by DNA - based duplication (the copying and pasting of DNA sequence from one genomic region to another) or retroposition. While DNA-based duplications are often tandem (Thornton 2007) , retroposed genes most often move to a new genomic environment, where they must acquire new regulatory elements or risk becoming processed pseudogenes (Kaessmann et al., 2009). The second mechanism is the alteration of existing gene structures. New gene structure can be generated by modifying existing exons or domains. Gilbert (1978) proposed that exons and domains could be recombined to produce new chimeric genes. In addition, retroposed sequences may jump into or near existing genes and recruit existing exons, or be recruited into an existing coding sequence (Zhang et al., 2009). Xue et al. (2003) found that Epstein-Barr virus contains an early gene which undergoes frequent frameshifts, probably to combat host immunity. In addition, divergenece in alternative splicing patterns between duplicate genes can generate distinct transcripts that produce noncoding RNA or polypeptides with slightly orentirely different functions and rapidly alter duplicate gene structures and functions (Zhang et al., 2010 : Zhou et al., 2011 ).

The third mechanism is De novo gene. New gene structures may arise from previously non-coding DNA. Chen et al.(1997) were the first to show that antifreeze protein, which bind and halt the growth of ice crystals in the blood of some polar fishes, were created byamplification of microsatellite DNA.
Since then a number of de novo genes originating from non-coding regions have been identified in human (Xie et al., 2012). Another origen of de novo gene is the horizontal gene transfer (HGT), whereas exchange of genes between genomes from distantly related taxa can immediately add new genes and functions in a genome. HGT is a major mechanism for the addition of new genes to prokaryotic genomes (Koonin et al., 2001), but has also been reported in a number of eukaryotic organisms including plants (Yoshida et al., 2010), insects (Moran et al., 2010), and fungi (Hall et al., 2005). Host-parasite interaction is one type of relationship usually pointed in the literature that could potentially increase the probability of the horizontal transfer between species, because the species involved in such relationships are generally in close contact. Transposable elements, which are well-known genomic DNA , are DNA entities that tend to be involved in horizontal transfer due to their ability to mobilize between different genomic locations (Ortiz et al., 2014). Movement of RNAs between cells of a single plant is well documented. Kim et al. (2014) sequenced transcriptomes of Cuscuta growing on Arabidopsis and tomato hosts to characterize mRNA transfer between species and found that mRNA move in high numbers and in a bidirectional manner. These findings demonstrate that parasitic plants can exchange large proportions of their transcriptomes withhosts, providing potential mechanisms for RNA-based interactions between species and horizontal gene transfer.Feng et al. (2014) found and suggest that sequence diversity of tRNA-linked STR in E. nuttalli occurs with relatively highly frequency and might be marker of geographical distribution of host rhesus macaques, even in 
limited area. This result showed the effect of geographical The fourth mechanism for production of new genes is noncoding RNAs. Not all new genes code for proteins. Noncoding RNAs were found to play an important role in neuronal functions in the early 1990s (Toll-Riera et al., 1993). Dai et al., (2008) showed that a new long noncoding RNA influences courtship behavior in $D$. melanogaster.

The fifth mechanism is transposable elements (TE). The genomic DNA is dynamic and not static, this proofed by transposable elements and horizontal gene transfer between intimate contact.TEs can mediate gene recombination by carrying coding sequences from one part of the genome to another (Yang et al., 2008). In addition, TEs were found to be a source of micro-RNAs, major components of posttranscriptional regulation of expression (Wang et al., 2012). Mobile elements are DNA sequences that can change their position (retrotranspose) with the genome. It has long been thought that neuronal genomes are invariable ; however recent studies have demonstrated that mobile elements actively retrotranspose during neurogenesis, thereby creating genomicdiversity between neurons. In addition, mounting data demonstrate that mobile elements are misregulatetrd in certain neurological disorders, including Rett syndrome and schizophrenia (Erwin et al., 2014).Transposable elements have an ongoing, largely parasitic interaction with their host. Hellen and Brookfield (2013) examined the sequence divergence between class II DNA transposons from mammalian genomes. They reported that, these sequences undergo a continuing process of turnover, keeping a family as an integrated whole, as members of the family are continually created and lost. Gifford et al. (2013) reported that, TEs play an important role in somatic tissues and evolution.

\section{Viruses and evolution}

Viruses consider the main source for evolution through integration into the genome of organisms from bacteria to human. About $5 \%$ of the human genome sequence is composed of the remains of rtroviruses that over millions of years have integrated into the chromosomes of egg and /or sperm precursor cells. There are indications that protein expression of these viruses is higher in some diseases. (Marchi et al., 2014). Human papillomavirus (HPV) integration is a key genetic event in cervical carcenogenesis.By conducting whole-genome sequencing and high throughput viral integration detection $\mathrm{Hu}$ et al. (2015) identified 3.667 HPV integration breakpoints in 26 cervical intraepithelial neoplasias. Moreover microhomologous sequence between the human and HPV genomes was significantly enriched near integration breakpoints, indicating that fusion between viral and human DNA may have occurred by microhomology-mediated DNA repair pathways. (Hu et al., 2015). Human papilloma virus (HPV) integration within the E2 gene has been proposed as a critical event in cervical carcenogenesis. The radiation therapy caused an eight-fold increase in the risk of HPV16 genome disruption. The integration status is influenced by the irradiation modalities, interestingly E2disruption being found widely after radiotherapy variation on the diversity of tRNA or genetic materials. treatments with a total fractioned dose of $50 \mathrm{~Gy}$. (Kahla et al., 2014).

Approximately $1 \%$ of human population inherits a chromosomally integrated copy of human herpesvirus 6 (C1-HHV-6). Huang et al. (2013)detected extrachromosomal circular HHV-6 molecules and they found that truncated C1-HHV-6 and extra-chromosomal circular molecules are likely reciprocal products that arise through excision of telomere-loop (t-loop) formed within the CIHHV-6 genome. After release of viral sequences from human telomere as circular molecules, some of which have the potential to become fully functioning viruses (Huang et al. ,2013)

Integration of the viral DNA into host chromosomes was found in most of the hepatitis B virus (HBV)- related hepatocellular carcinomas (HCCs ). HBV integration favored chromosome 17 and preferentially integrated into human transcript units. Ding et al. (2012) identified 8 genes that were recurrent target genes by $\mathrm{HBV}$ integration including fibronectin 1 (FN 1) and telomerase reverse transcriptase (TERT 1), two known recurrent target genes, and additional novel target genes such as SMAD family member 5 (SMAD 5), phosphatase and actine regulator 4 (PHACTR4), and RNA binding protein fox-1 homolog (G. elegance) 1 (RBFOX1). Moreover , Ding et al. (2012) identified 14 additional recurrent HBV target genes (Ding et al., 2012)).

Jacobs et al. (2014) reported that throughout evolution primate genomes have been modified by waves of retrotransposon insertion. Kvaratskhelia et al. (2014) Retroviral replication proceeds through an obligate integrated DNA provirus making retroviral vectors attractive vehicles for human gene -therapy. Though most of the host cell genome is available for integration, the process of integration site selection is not random. Lentiviruses including HIV-1 preferentially integrate within the bodies of active genes, whereas the prototypical gammaretrovirus Moloney murine leukemia virus (MoMLV) favors strong enhancers and active gene promoter

\section{Bacteria and evolution}

Bacteria also, chair in the evolution through transfer of genetic materials to its host. Natural competence for transformation is a developmental program that allows certain bacteria to take up free extracellular DNA from the environment and integrated this DNA into their genome. Thereby, natural transformation acts as mode of horizontal gene transfer and impacts bacterial evolution. (Matzger and Blokesch , 2014). Horizontal gene transfer (HGT) is a major driving force of bacterial evolution. The rapid exchange of genetic information mediated by HGT enables bacteria to adapt to new environmental niches, to spread harmful traits such as antibiotic resistance cassettes or pathogenicity island and to maintain genome integrity (Stokes and Gillings. , 2011). Horizontal gene transfer describes the transmission of genetic material across species boundaries. HGT often occurs in microbic and eukaryotic genomes. However, the pathways by which 
HGTs occur in multicellular eukaryotes, especially in plants, are not well understood. Gao et al. (2014) systematically summarized more than ten possible pathways for HGT. The intimate contact which frequently occurs in parasitism, symbiosis, pathogen, epiphyte, entophyte, and grafting interactions couldpromote HGTs between two species. Besides these direct transfer methods, gene can be exchanged with a vector as a bridge , possible vectors include pollen, fungi, bacteria, viruses, viroids, plasmids, transposons, and insects. HGT, especially when involving horizontal transfer of transposable elements, is recognized as a significant force propelling genomic variation and biological innovation, playing an important fuctional and evolutionary role in both eukaryotic and prokaryotic genomes(Gao et al. ,2014). Wang et al., 2007 demonstrated intergenus natural genetic transformation between Escherichia coli and Bacillus subtillus at different growth phase.

\section{RNA and evolution}

Short interspersed elements (SINEs) act as driving forces in genome evolution (Schmitz , 2012). SINEs are short interspersed elements derived from cellular RNAs that repetitively retropose via RNA intermediates and integratemore or less randomly back into the genome. SINEs propagated almost entirely vertically within their host cells and ,once estaplished in the germline, are passed on from generation to generation. As non-autonomous elements, their reverse transcription (from RNA to cDNA) and genomic integration depends on the activity of the enzymatic machinery of autonomous retrotransposens, such as long interspersed elements (LISEs). SINEs are widely distributed in eukaryotes, but are especially effectively propagated in mammalian species. More than a million Alu-SINE copies populate the human genome (approximately $13 \%$ of genomic space), and few master copies of them are still active. SINEs are served as beneficial building blocks for evolution, contributing to phenotypic heterogeneity and modifying gene regulatory networks (Schmitz, 2012). They substantially expand the genome space and introduce structural variation to the genome. SINSEs have the potential to mutate genes, to alter gene expression, and generate new parts of genes. A balanced distribution and controlled activity of such properties is crucial to maintaining the organism's dynamic and thriving evolution (Schmitz, 2012).

\section{Nutrition and micro-RNA}

Environment transfer to organisms through food (nutrition), drinking and air (respiration), all previous parameters has magnified effects on genetic materials producing variations of living organisms through induction of benefit or harmful mutations, or variation of gene expression or appears of de novo gene from non-coding DNA.Eating is an engagement with the world, that transfer our environment into our bodies (Pollan , 2006). Experimental study reported that micro-RNAs (miRNAs) from plants may control target genes in the consumer (Zhang et al. , 2012). Food-derived plant miRNA entered mammalian circulation naturally and achieved levels comparable to those of abundant endogenous extracellular miRNA. Plant micro-RNAs were detected not only in circulation, but also in all murine tissue types examined, and at copy numbers rivaling those of endogenous miRNAs. In liver, some plant miRNA were more abundant than let-7a. In the mouse, plant miRNA were said to downregulate at least one endogenous target in liver, the low-density lipoprotein receptor adapter protein one 1 (LDLRAPI), within hours of dietary intake (Kenneth and Kendai , 2014)

Snow et al. ( 2013) reviewed non-dietary means of systemic xenomiRNA transfer. Organ or stem celltransplants, blood transfusions, pregnancy, and various states of parasitismmay involve direct RNA transfer from one organism to another. Inhalation of atmospheric plant material, injection of plant-based drugs, and fluid exchanage (e.g. through different sexual practices) could allow RNA transfer.

A different sort of extracellular RNA (exRNA)-mediated communication may exist in some eukaryotes (Witwer et al.,2013). RNA molecules associated with several extracellular carriers, including lipoprotein (Vickers et al., 2011) and protein complexes (Turchinovich and Burwinkel , 2012), but extracellular vesicles (EV) have enveloped viruses and EV have been examined (Wurdinger et al., 2012)

We developed in our lab electrophoretic pattern of nucleic acid technique, which is based on gently squeezimg of solid tissue with blue tips and directly run in electrophoresis. This technique reflect the state of DNA and RNA without any technical or mechanical degradation. Surprisingly, we detected RNA less than 50 bp we predict that this RNA may be micro-RNA (Fig. 4). The technique of sister chromatid exchange (SCE) reflect the mutation and instability of genome (Figs. 5, 6, 7) through increasing the rat of frequency of SCE than the normal rate. We observed some environmental pollution and drugs increased this rate, this reflect the effect of environmental pollution for increasing the rate of transfer or movement of transposable elements and induction of variation in somatic or germinal genome. We found individual variation in one species of Artemia sp. and eel Anguilla Anguilla using haplotype technique (Fig. 8).

\section{References}

Arroyo J.D., Chevillet J. R., Kroh E. M., Ruf I. K., et al.,(2011). Argonaute2 complexes carry a population of circulating microRNAs independent of vesicles in human plasma. Proc Natl Acad Sci USA. 2011;108:5003-8.

Chen ,L.. ,DeVries A. L. and Cheng C.H. (1997). Evolution of antifreeze glycoprotein gene from a trypsinogen gene in Antarctic notothenioid fish. Proceedings of the National Academy of Sciences of the United States of America . ; 94(8) : 3811-3816

Dai H., Chen, Y., Chen S. Mao Q ., Kennedy D.;Landback, P.;Eyre-Walker A.;Du, W. and Long M. (2008). The evolution of courtship behaviors through the origination of a new gene in Drosophila. Proceedings of the National Academy of Sciences of the United States of America. 105(21) ; 7478-7483. 


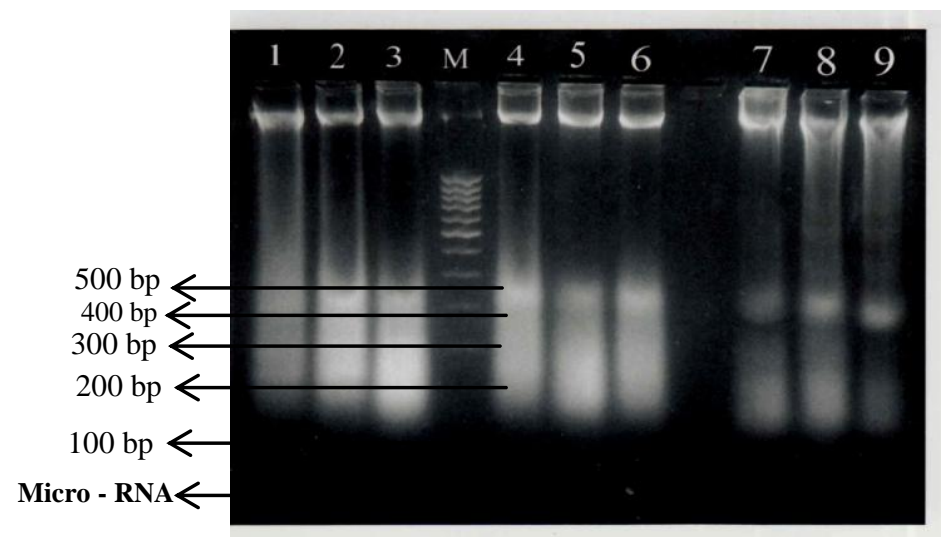

Fig. (4) Micro- RNA less than 100 bp (after Hassab El-Nabi, 2004 )

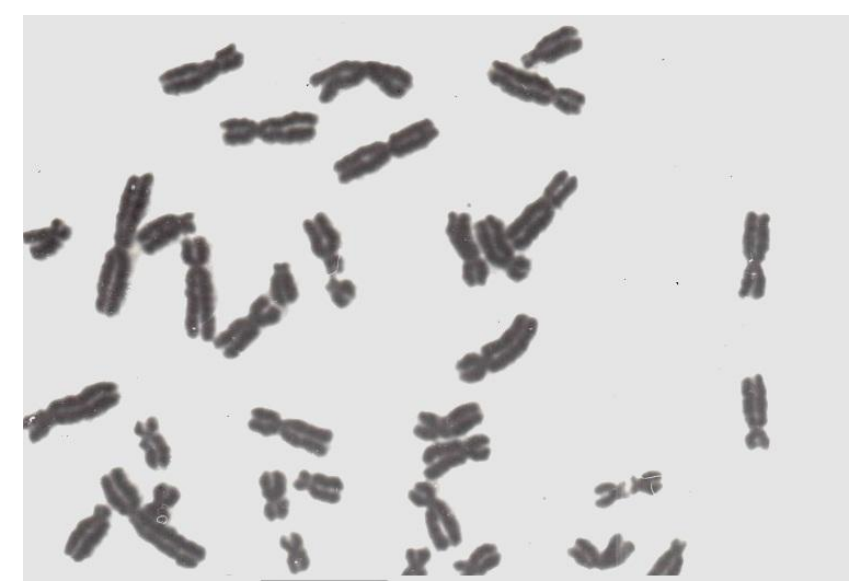

Fig. (5) Normal human metaphase (after Hassab El-Nabi, 1994 )

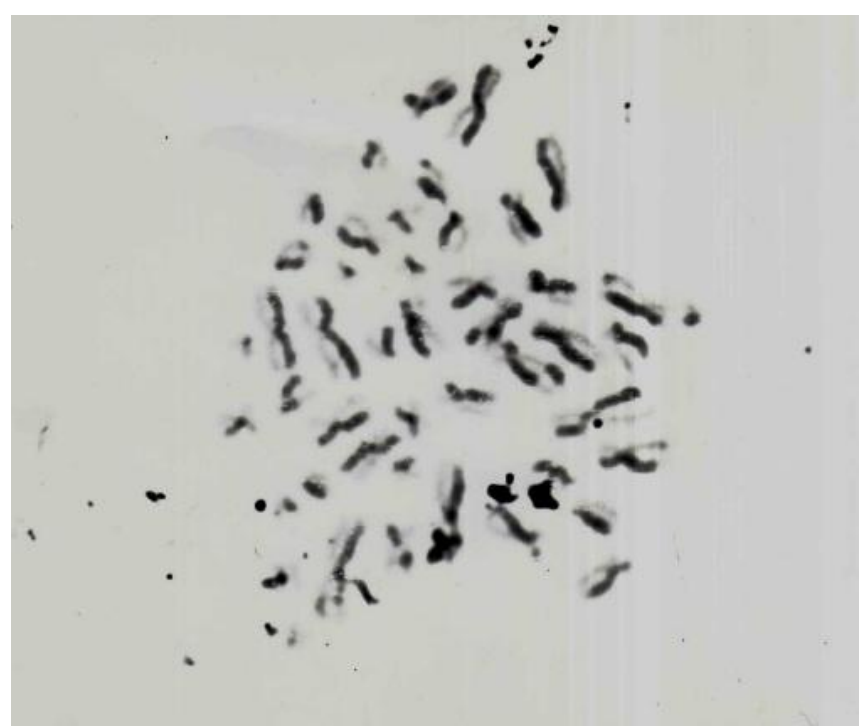

Fig. (6) Sister chromatid exchanges (SCE) in control human metaphase (after Hassab El-Nabi, 1994 ) 


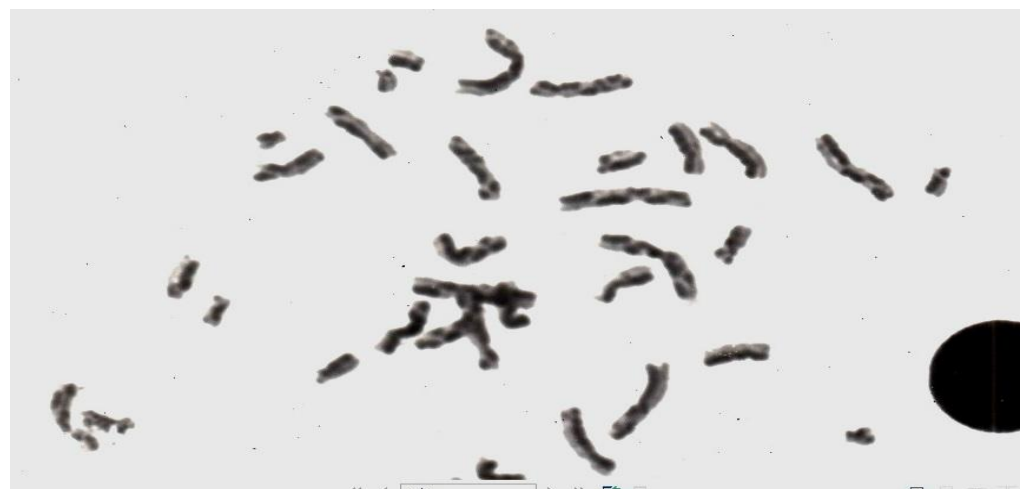

Fig. (7) Sister chromatid exchanges (SCE) in treated human metaphase with mitomycin C (after Hassab El-Nabi, 1994 )

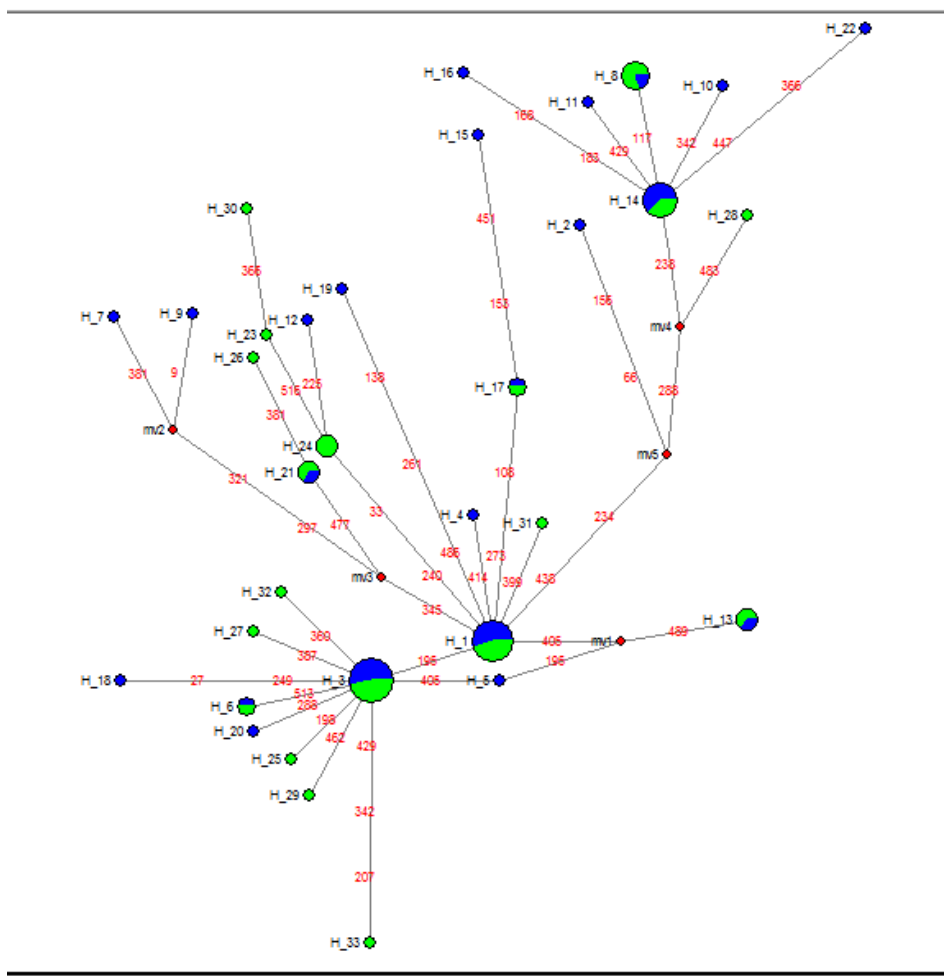

Fig. (8).Different haplotype of eel Anguilla anguilla (after Hassb El-Nabi et al., 2016)

Ding D., Lou X., Hua D., Yu W., Li L., Wang J., Gao F., Zhao N., Ren G., Li l. and Lin B. (2012).Recurrent targeted genes of hepatitis $\mathrm{B}$ virus in the liver cancer genomes identified by a next-generation sequencing-based approach. PLoS Genet. 8(12): e1003065.

Edvotec (2014). The genesis of new genes ;part 2: The Biotechnology Education company.

Erwin J. A., Marchetto M. C. and Gage F. H. (2014). Mobile DNA elements in the generation of diversity and complexity in the brain. Nat. Rev. Neurosci . 15(8): 497506.

Gao C., Ren X., Mason A. S., Liu H., Xiao M., Li J. and Fu D. (2014).Horzontal gene transfer in plants. Funct. Integr. Genomics ; 14(1): 23-29.
Gifford W. D., Pfaff S. L. and Macfarlan T. S. (2013). Transposable elements as genetic regulatory substrates in early development. Trends Cell Biol. 23(5): 218-226.

Hassab El-Nabi S.E., Mohammed-Geba K., and Saied M. (2016). Genetic population structure of eel Anguillo Anguilla in lack Burullus and Rachid estuary (in press)

Hassab El-Nabi, S. E. (2004). Molecular studies on the relationship between apoptosis and DNA damage induced by dexamethasone and flumox in liver and spleen of rat and human lymphocytes culture. J. Egypt. Ger. Soc. Zool. (45 C): Histology \& HistochemiStry, 175 - 202. 
Hassab El-Nabi S.E, (1994). Genetic monitoring studies on selective environmental pollutants (pesticides) suspected to be mutagens to mammalian animals. Ph.D. Thesis ; Zoology departments, Faculty of Science, Menoufiya University

Hecht M.M., Nitz N., Araujo P. F., Sousa A. O., Rosa Ade C., Gomes D. A., Leonardecz E. and Teixeira A. R. (2010). Inheritance of DNA transferred from American trypanosomes to human hosts. PLoS one; 5(2): e9181.

Hellen E. H. and Brookfield J. F. (2013).Transposable element invasions. Mob Genet Elements 3(1): e23920.

Hu Z., Zhu D., Wang W., Li W., et al. (2015). Genome-wide profiling of HPV integration in cervical cancer identifies clustered genomic hot spots and a potential microhomology-mediated integration mechanism. Nat. Genet.; 47(2): 158- 163.

Huang Y., Hidalgo-Bravo A. Zhang E., Cotton V. E., Mendez-Bermudez A., Wig G., Medina-Calzada Z., Neumann R., Jeffreys A. L., Winn B., Wilson J. F., Clark D. A., Dyer M. J. and Royle N. J. (2014). Human telomeres that carry an integrated copy of human herpesvirus 6 are often short and unstable, facilitating release of the viral genome from the chromosome. Nucleic Acids Res. ; 42(1): 315-27.

Jacobs F. M., Greenberg D., Nquyen N., Haeussler M., Ewing A. D., Katzman S., Paten B., Salam S. R. and Haussler D. (2014). an evolutionary arms race between KRAB zing-finger genes ZNF91193 and SVAlL1 retrotransposens. Nature; 516(7530): 242-5.

Kaessmann $\mathrm{H}$, Vinckenbosch $\mathrm{N}$, and Long $\mathrm{M}$ (2009). RNA-based gene duplication: mechanistic and evolutionary insights. Nature Reviews Genetics. 10(1): 1931.

Kahla S., Kochbati L., Maalei M. and Queslati R. (2014). Situation of HPV16 E2 gene status during radiotherapy treatment of cervical carcinoma. Asian Pac J. Cancer Prev. 15(6): 2869-73.

Kenneth W. Witwer and Kendal D. Hirschi (2014). Transfer and functional consequences of dietary microRNAs in vertebrates: Concepts in search of corroboration Bioessays.; 36(4): 394-406.

Kim G.,LeBlanc M. L., Wafula E. K., dePamphilis C. W. and Westwood J. H. (2014). Plant science, Genomicscale exchange of Mrna between a parasitic plant and its host. Science, 15;345(6198) : 808-811.

Kuraku S., Qiu H. and Meyer A. (2012). Horizontal transfers of Tc1 elements between teleost fishes and their vertebrate parasites , lampreys. Genome Biol. Evol.; 4(9): 929-936.

Kvaratskhelia M., Shama A., Larue R. C., Serrao E. and Engelman A. (2014). Molecular mechanisms of retroviral integration site. Nucleic acids Res.; 42(16): 10209-25.
Manyuan Long , Nicholas W. Vankuren , Sidi Chen and Maria D. Vibranovski (2013).New gene evolution: little did we know , Annu. Rev. Genet. 47 ; 307-333

Marchi E., Kanapin A., Magiorkinis G. and Belshaw R. (2014). Unfixed endogenous retroviral insertions in the human population. J. Virol.; 88(17): 9529-37.

Metzger L.C. and Blokesch M. (2014). Composition of the DNA-uptake complex of Vibrio cholera. Mob. Genet. Elements; 4(1): e28142.

Ortiz M. F., Wallau G. L. , Graichen D. A. and Loreto E. L.(2014). An evaluation of the ecological relationship between Drosophila species and their parasitoid wasps as an opportunity for horizontal transposon transfer. Mol. Genet. Genomics . 290(1): 67-78.

Pollan M. (2006). The Omnivore's Dilemma: A Natural History of Four Meals. New York: Penguin Press; 2006.

Schmitz J. (2012). SINEs as driving forces in genome evolution. Genome Dyn. 7 : 92-107.

Sidi Chen,Benjamin H. KrinskyManyuan Long (2013). Box 1: Mechanisms of new-gene origination;Nature Reviews Genetics; 14: 645-660

Stokes H. W. and Gillings M. R. (2011). Gene flow, mobile genetic elements and the recruitment of antibiotic resistance genes into Gram-negative pathogens. FEMS Microbial Rev.; 35: 790-819.

Thornton K.R. (2007): The neutral coalescent process for recent gene duplications and copynumber varients. Genetics, 177(2): 987-1000

Tiedge H.,Chen W. and Brosius J.(1993). Primary structure, neural-specific expression, and dendritic location of human BC200 RNA. The Journal of Neuroscience. 13: 2382-2390.

Toll-Riera M., Bosch N., Bellora N.,Castelo R., Armengol L., Estivill X.and Alba M. M. (2009).Origin of primate orphan genes: A comparative genomics approach. Molecular Biology and Evolution. 26(3): 603-612.

Turchinovich A. and Burwinkel B. (2012). Distinct AGO1 and AGO2 associated miRNA profiles in human cells and blood plasma. RNA Biol. ;9:1066-75.

Vickers K. C., Palmisano B.T., Shoucri B. M., Shamburek R.D., et al.(2011). MicroRNAs are transported in plasma and delivered to recipient cells by high-density lipoproteins. Nat Cell Biol. ;13:423-33.

Witwer K. W., Buzas E. I., Bemis LT, Bora A, et al. (2013).Standardization of sample collection, isolation and analysis methods in extracellular vesicle research. J Extra cell Vesicles.2013;2 10.3402/jev.v2i0.20360.

Wurdinger T., Gatson N.N., Balaj L., Kaur B., et al.,(2012). Extracellular vesicles and their convergence with viral pathways. Adv Virol. 2012:1-12

Xie C., Zhang Y. Y., Chen J-Y., Liu C-J., Zhou WZ., Li Y., Zhang M., Zhang R., Wei L. and Li C-Y (2012). Hominoid-specific De Novo protein-coding genes 
originating from long non-coding RNA . PLoS genetics. 8(9): e1002942 .

M. D., Lu Q.,Middeldorp J. M. and Griffin B. E.(2003). Genetic diversity : Frameshift mechanisms alter coding of a gene (Epstein -Barr Virus LF3 gene) that contains multiple 102-base pair direct sequence repeats . Molecular and Cellular Biology. 23(6): 2192-2201.

Yang S., Arguello J. R., Li X., Ding Y., Zhou Q., Chen Y. and Zhang Y., et al (2008). Repetitive elementmediated recompination as a mechanism for new gene origination in Drosophila. PLoS Genetics. 4 (1): e3.

Yoshida S., Maruyama S., Nozaki H. and Shirasu K. (2010). Horizontal gene transfer by the parasitic plant striga hermonthica. Science. $328: 1128$.

Zhang L. , Hou D., Chen X., Li D., et al., (2012).Exogenous plant MIR168a specifically targets mammalian LDLRA P1: evidence of cross-kingdom regulation by microRNA. CellRes. ;22:107-26.

Zhang P. G., Huang S. Z. Pin A-L and Adams K. L. (2010). Extensive divergence in alternative splicing patterns after gene and genome duplication during the evolutionary history of arsbidopsis. Molecular Biology and Evolution. 27 (7): 1686-1697.

Zhang Y., Lu S., Zhau S., Zheng X., Long M.and Wei L. (2009). Positive selection for the male functionality of a co-retroposed gene in the hominoids. BMC Evolutionary Biology. 9 : 252.

Zhou R., Moshgabadi M. and Adams K. L.(2011). Extensive changes to alternative splicing patterns following allopolyploidy in natural and resynthesized polyploids. Proceeding of the National Academy of Sciences of the United States of America. 108(38): 16122-16127. 